\title{
Strategy to Develop Video Platform Under the Perspective of Media Convergence Based on a Comparative Study
}

\author{
Shuyue Li \\ School of Economics and Management, Beijing Jiaotong University, Beijing, China \\ Email: shuyueli2020@163.com
}

\begin{abstract}
Keywords: media convergence, video platform, China Blue Platform, Mango TV
\end{abstract}
\begin{abstract}
Under the background of media convergence, a number of television stations respond to national policies to build video websites or platforms to provide video services to audiences. Zhejiang Television Station and Hunan Television Station launched China Blue platform and Mango TV respectively, but their performance is quite different. Therefore, this paper hopes to provide countermeasures and suggestions for the development of video platform by analyzing the two kinds of media convergence models.
\end{abstract}

\section{Introduction}

With the rapid development of Internet and new media technology, people's living habits have been changed. They tend to watch programs and get news through the Internet. Based on this, the Chinese government has vigorously promoted the convergence of radio and television media and emerging media, to provide better services for people, as well as to alleviate the impact on traditional television stations. Television stations respond to national policies, from the construction of websites to the development of mobile clients, from building platforms to relying on platforms.[1] They achieve development through continuous service innovation.However, due to the difference of status and production ability between television stations, their strategies are quite different. For local television stations, because of the constraints of capital and talents, they are more likely to provide video services through the basic website construction, and to disseminate news and information through new media clients. However, the wide use of mobile phones makes it quite difficult to attract users for them. And now, users' demand for video is mainly for entertainment. At present, only a few TV stations have established their own platform to provide video services to users, such as China Blue platform set up by Zhejiang Television Station and Mango TV set up by Hunan Television Station. However, both relying on the support from television stations, there is a large gap between Chinese Blue Platform and Mango TV. Mango TV has been able to compete with video platform such as Youku and Iqiyi, and even have a better performance than them in terms of profitability. While Chinese Blue Platform has not obtained the better development. So what is the reason for this kind of difference? What efforts should be taken to help the development of video platform established by television station? Therefore, the paper chooses China Blue Platform and Mango TV as the research objects, hoping to provide countermeasures and suggestions for the platform to attract users to enhance their competitiveness.

\section{The Concept of Media Convergence}

In 2010, the State Council proposed to promote the development of three media convergence. It is defined as the connect among telecommunications network, radio and television network, and Internet. They share resource and provide users with voice, data, radio and television as well as other services. Based on this, the concept of media convergence is put forward, which is considered to be the convergence of television stations and the Internet. It is a mode in which the traditional radio and television media complement and develop through the Internet and then provide services to users. [2] And under today's environment, it specifically refers to radio and television stations providing services to users in the form of common development with the platform.[3] 


\section{The Strategy of Video Platform Under the Background of Media Convergence}

\subsection{The Strategy of Chinese Blue platform}

In terms of the distribution of resources, Zhejiang Television Station is the main source for Chinese Blue platform to obtain videos, so the capability of Zhejiang Television Station are of great importance for the development of video platform. However, a part of Zhejiang Television Station's videos made by itself are purchased by other video platform, and the outside-purchased video resources are authorized to play on the Chinese blue platform, so Chinese Blue Platform is short of video copyright.

From the point of view of products and services, Chinese Blue platform provides video broadcast from all channels of Zhejiang Television Station, as well as some derivative videos of its famous programs. By building a discussion community, it attracts some fans of its famous programs. And fans can also get unique identity authentication and high-definition video experience after being member.

The revenue of China Blue Platform comes from users' purchase for member.

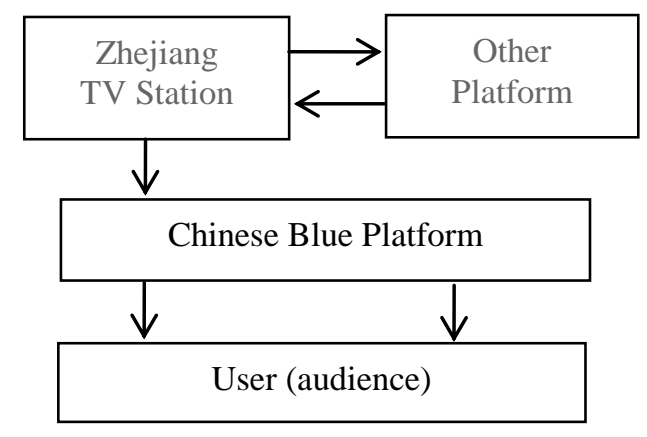

Fig. 1 The Platform Strategy of Chinese Blue Platform

\subsection{The Strategy of Mango TV}

In terms of the distribution of resources, Mango TV is the only channel for users to watch the particular videos from Hunan Television Station. At the same time, it also makes its own hot programs and outputs them to Hunan Television Station in reverse. In addition, Mango TV also purchase video resources from outside.

From the point of view of products and services, Mango TV provides users with a variety of services, including popular videos, novels, listening service and so on. By providing multiple programs and other services, it nearly meet the diverse needs of all age groups. The users can be readers, audiences and other identities. Through the construction of interactive community, it master the entertainment needs of users, user also participate in production. Membership services allow users to access film and television resources in advance, avoiding watching advertisements.

On one hand, the revenue of the Mango TV comes from the uses' multiple purchase behavior. Audiences purchase member for video watching, while reader pay for novels. On the other hand, as a result of the diversity of the platform resources and the huge customer base, Mango TV has attracted many advertisers. They choose to sponsor the programs play on the platform. 


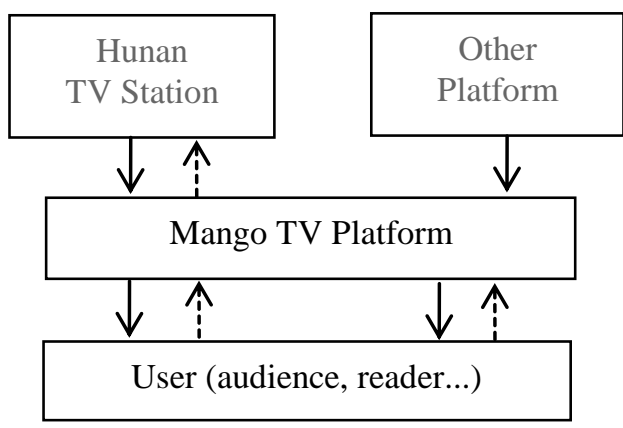

Fig. 2 The Platform Strategy of Mango TV Platform

\subsection{A Comparative Analysis between Chinese Blue Platform and Mango TV}

We know that there is a big gap between the user base and profit model of two platform. And through the analysis of the strategies of Chinese Blue Platform and Mango TV, we find the main causes are as follows.

The relationship between the television station and the video platform affects the video resource of platform. The development of Chinese Blue Platform almost totally depend on the unilateral export of video from television station. While Mango TV can obtain resource from outside, and it also export video resources to television station. Besides, the television station doesn't only provide Chinese Blue Platform with its video resources, while Mango TV obtain all the video resources from television station. Thus, there is a big gap between the number of videos, and users' seeking for more resources affect their choice of the video platform.

The value proposition of the video platform affects the user base. In terms of Chinese Blue Platform, it is just like a surface of television station, live broadcast doesn't fit for every user. And only by providing live broadcast service, it can not satisfy user's other demand. However, Mango TV is a gather of all the entertainment products, it can also attract users with other identities. And user base also affects the investment. In addition because of the different value proposition, the efforts they put on the development of video platform is also different, which affect their information from users, and then influence their new product development.

Above all, we think the way of media convergence is quite different between two platform. The strategy of Chinese Blue Platform is a traditional way of media convergence, while the strategy of Mango TV is a new way of media convergence. And under the development of Internet, the traditional one should turn to the new one, from a simple "you provide broadcast to me" model to a user-driven television program change.[4]

\section{Countermeasures and Suggestions for the Future Development of Video platform}

Under the background of media convergence, the development of the video platform need the effort made by both television stations and video platforms.

\subsection{Television stations}

Under the background of media convergence, video platform is an important carrier for television stations to attract users and improve profit level. Thus television stations should vigorously support the development and construction of video platform. First, as the main video provider of the platform, the television stations should improve its production capacity by investigating users' demand, especially the demand of young users. And then users will prefer to use their video platform to watching videos. Second, television stations should make contact with more film and television companies to guarantee the variety of the video. Beside it is also important for television stations to send their particular video resource to video platform to avoid the loss of core fans. 


\subsection{Video platforms}

Besides as the complementary to the television stations, on the one hand, video platforms should provide a variety of services for the users, on the other hand, it should provide information for the program production of the television stations by obtaining the needs of the users. The video platform should improve its production ability, through the user behavior obtained from the platform to analyze users' preferences for content, and then select the project to develop. Providing users with the opportunity to communicate through the construction of the network community and the provision of other network services is also effective. In addition, video platform can be the interface for other Internet services, such as novels and online shopping to increase sources of income. And after that, they can purchase programs from other platform and film and television company, and reduce their dependence on television station.

\section{Summary}

Under the background of media convergence, it is an inevitable trend for television stations to develop through the establishment of video platform. Through the comparative analysis of Mango TV and Chinese Blue Platform, we found that the proposition and ability of the platform, the relationship between the platform and the television stations will have an impact on the user base and profit model of the video platform. Based on this, we give some countermeasures for the future development of video platform.

\section{References}

[1] Tang Yan, Bo Hanbin. Network Integration Strategy in the all-Media era-taking Hunan Satellite TV, Mango TV as an example [J]. New Media Research, 2018, 4 (07): 96-97

[2] Zhang Zinuo. Research on the Strategies of Dalian Radio and TV Station to Network Convergence [D]. Dalian University of Technology, 2016.

[3] Song Zhijian. Research on Wenzhou Construction Mode of all-Media Integrated production platform [J]. Television Technology, 2017, 41 (Z4): 115-118.

[4] Xu Shanwen. The research of the integration business model of the China network in the Web3.0 era[D]. Graduate School of Chinese Academy of Social Sciences,2014. 